\title{
EFEITO DA MOTIVAÇÃO DOS ACADÊMICOS DE CIÊNCIAS CONTÁBEIS SOBRE AS ESTRATÉGIAS DE APRENDIZAGEM ADOTADAS POR ELES: ESTUDAR OU COMPREENDER?
}

\section{STUDY OR UNDERSTAND? THE EFFECT OF THE ACADEMIC MOTIVATION OF ACCOUNTING SCIENCES ON THE LEARNING STRATEGIES ADOPTED BY THEM}

“O artigo foi aprovado e apresentado no ENANPAD 2018, realizado de 03/10 a 06/10 de 2018, em Curitiba”

\begin{abstract}
Resumo
Os níveis motivacionais dos alunos é um assunto tratado com afinco no contexto educacional, tanto como elemento de reflexão na forma de ensinar, como no modo de aprender. A Teoria da Autodeterminação defende que o nível de motivação dos acadêmicos produz efeitos no emprego de estratégias de aprendizagem. Assim, esse estudo verificou o efeito da motivação sobre as estratégias de estudo adotadas pelos acadêmicos de Ciências Contábeis. Realizou-se uma survey com 106 acadêmicos de uma universidade pública do Paraná. Na análise dos dados empregou-se a técnica de análise fatorial e regressão múltipla. Os achados confirmam o efeito da motivação na forma de estudar. Observou-se que as motivações de ordem intrínseca se relacionaram positivamente a dedicação e a adoção de estratégias de estudo aprofundadas. Adicionalmente, confirmou-se também que o emprego de estratégias de estudos operacionais é estimulado por pressões/motivações externas. Os achados contribuem significativamente para a compreensão dos diversos estímulos que atuam sobre a forma de aprendizagem adotada pelos discentes ao longo de sua vida acadêmica. Além disso, estimula a compreensão social do comportamento dos discentes no processo de ensino e aprendizagem dos estudantes ao oferecer conhecimento sobre a sua motivação.
\end{abstract}

Palavras-chave: Motivação. Estratégia de aprendizagem. Teoria da Autodeterminação. Acadêmicos de Ciências Contábeis.

\begin{abstract}
The motivational levels of the students is a subject dealt with hard in the educational context, as much as reflection element in the way of teaching, as in the way of learning. The Self-Determination Theory argues that the motivation level of academics produces effects on the use of learning strategies. Thus, this study verified the effect of motivation on the study strategies adopted by accounting students. A survey was conducted with 106 students from a public university in Paraná. In the data analysis we used the technique of factor analysis and multiple regression. The findings confirm the effect of motivation on the way we study. The intrinsic motivations were positively related to the dedication and adoption of in-depth study strategies. Additionally, it was also confirmed that the use of operational study strategies is stimulated by external pressures / motivations. The findings contribute significantly to the understanding of the various stimuli that act on the form of learning adopted by students throughout their academic life. In addition, it stimulates the social understanding of student behavior in the teaching and learning process of students by providing knowledge about their motivation.
\end{abstract}

Keywords: Motivation. Learning strategy. Self-Determination Theory. Academics of Accounting Sciences.

\begin{abstract}
Franciele do Prado Daciê
Doutoranda em Administração pela Universidade Estadual de Maringá (UEM). Mestre em Contabilidade pela Universidade Federal do Paraná (UFPR). Especialista em Controladoria e Finanças pela Universidade Estadual de Maringá (UEM). Bacharel em

Ciências Contábeis pela Universidade Estadual de Maringá (UEM). Docente na Universidade Estadual de Maringá (UEM). Contato: Rua Dom Pedro II, 598, zona 01, Cianorte, PR, CEP 87.200-055. E-mail: frandacie@gmail.com.
\end{abstract}

\section{Marcielle Anzilago \\ Doutora em contabilidade pela Universidade Federal de Santa Catarina (UFSC). Mestre em contabilidade pela Universidade Federal do Paraná (UFPR). Graduada em Ciências Contábeis pela Universidade Estadual do Mato Grosso do Sul (UEMS). Docente do curso de Ciências Contábeis na Universidade Federal do Mato Grosso do Sul (UFMS). Contato: Av. Rosilene, R. Eugenia Oliveira Lima, 64 - Bairro Universitário, Nova Andradina - MS, 79750-000, telefone (67) 3449-0500. Email: marcianzilago@gmail.com}




\section{INTRODUÇÃO}

A constante exigência de interpretação e reflexão diante da sociedade, mostra que a educação é fundamental para aumentar o nível da capacidade de agir dos indivíduos (Leal, Miranda \& Carmo, 2013). A educação institucionalizada por meio do ensino é a forma mais apropriada para formar um indivíduo na constituição da sua opinião, no contato com questões que estimulem o raciocínio crítico, criatividade e estímulo ao aprendizado (Leal, Miranda \& Carmo, 2013). Desse modo, o ensino superior, ambiente no qual ocorre a transmissão da experiência cultural e científica, torna-se um dos principais provedores do desenvolvimento econômico (Ferreira, 2009).

Acredita-se que a motivação dos alunos é um problema evidente no contexto educacional. Bzuneck (2005) observa que, de modo geral, os alunos ao final do ensino médio escolhem um curso superior por afinidade com a profissão, por influência dos pais ou, até mesmo por falta de opção. Ao entrar na universidade, os acadêmicos criam expectativas de que as disciplinas do cursos contribuirão significativamente para sua formação,e passam a designar aos professores a função de demonstrar como será essa contribuição (Bzuneck, 2005). No entanto, a literatura expõe que o progresso nas séries escolares é seguido por um declínio motivacional (Harter, 1981), ou seja, no início do curso existe uma maior motivação dos alunos em relação ao final dele (Oliveira et al., 2010).

O termo motivação, quando empregado ao contexto acadêmico, vincula-se ao estímulo, entusiasmo, interesse, vontade e prontidão ao estudo. Além disso, evidências apontam a existência de um relacionamento positivo entre aprendizagem e motivação dos estudantes (Boruchovitch \& Bzuneck, 2004). Nesse sentido, acredita-se que a motivação é indispensável para que os alunos se sintam empolgados a estudar, obtenham bons resultados, realizem-se academicamente e atinjam a satisfação em suas vidas (Lens, Matos \& Vansteenkiste, 2008).

Nesse aspecto, segundo a Teoria da Autodeterminação, a motivação pode ser oriunda de ordens intrínseca, extrínseca ou de desmotivação e, produz um efeito significativo no processo de ensino-aprendizagem dos estudantes (Deci \& Ryan, 1985; Ryan \& Deci, 2000b; Guimarães \& Bzuneck, 2008). Acredita-se que os tipos motivacionais podem exercer relações com as estratégias de aprendizagem adotadas pelos acadêmicos. Desse modo, a questão que motiva este estudo é: Qual o efeito da motivação dos acadêmicos de Ciências Contábeis sobre suas estratégias de aprendizagem?

Desse modo, esse estudo objetiva verificar o efeito da motivação sobre as estratégias de aprendizagem adotadas pelos acadêmicos do curso de Ciências Contábeis. Embora foram encontradas pesquisas internacionais que verificam a motivação e o nível estratégia de aprendizado (Vansteenkiste et al., 2004a; Vansteenkiste et al., 2005; Kusurkar et al., 2012; Munshi, Al-Rukban \& Al-Hoqail, 2012), uma lacuna foi observada quanto a análise das variáveis propostas em cenário brasileiro.

Sob a perspectiva da Teoria da Autodeterminação, estudos mostram que o tipo de motivação do indivíduo - intrínseca, extrínseca, desmotivação - pode afetar o desempenho, a criatividade e a autodeterminação em suas atividades (Deci \& Ryan 1991; Ryan \& Deci, 2000a; 2000b; Gagné \& Deci, 2005). Estímulos de ordem interna (intrínsecos) tendem a estimular proativamente as ações, enquanto aqueles derivados externamente relacionam a comportamentos mais reservados e negativos (Ryan \& Deci, 2000a; 2000b; Vansteenkiste et al., 2004a; Kusurkar et al., 2012). Nessa perspectiva, pesquisas brasileiras delimitam-se a avaliar os niveis de motivação entre disciplinas, entre períodos do curso e entre cursos com diferentes modelos de gestão (Guimarães et al., 2002; Oliveira et al., 2010; Lopes et al., 2015).

No entanto, esses estudos não apontam os efeitos que esses tipos motivacionais relacionam-se ao modo como os acadêmicos estudam/aprendem. Desse modo, este estudo avança em relação aos demais respaldados na Teoria da Autodeterminação, uma vez que analisa os efeitos que os tipos de motivação exercem sobre as estratégias de aprendizagem acadêmica. Portanto, entende-se que este estudo contribui no aspecto teórico sob a perspectiva acadêmica, mostrando como a motivação impacta na vida acadêmica dos estudantes, e permite uma compreensão social do comportamento dos discentes no processo de ensino e aprendizagem em cursos de nível superior. $O$ trabalho também contribui no âmbito do processo de ensino e aprendizagem dos estudantes ao oferecer conhecimento sobre a motivação dos acadêmicos.

A próxima seção apresenta a fundamentação teórica, na qual discorre sobre a Teoria da Autodeterminação, as motivações existentes nos indíviduos e as relações entre estratégias de aprendizagem e motivação dos alunos. Esses elementos tornaram-se necessários para fundamentar as hipóteses do estudo. A seção 3, por sua vez, apresentou os procedimentos metodológicos, ou seja, a amostra estudada, os métodos e análises aplicadas para atingir o objetivo do estudo. A seção 4 apresenta os resultados da pesquisa e discussões acerca deles. Por fim, a seção 5 consiste nas considerações finais do estudo.

\section{2 .REFERENCIAL TEÓRICO}

\subsection{Teoria da Autodeterminação}

A motivação é entendida como o sentimento que move um indivíduo, que o põe em ação ou o faz mudar de curso. Ela pode manifestar-se de forma diferente em cada um, levando-o a persistir de forma mais ou menos intensa na realização de uma tarefa (Boruchovitch \& Bzuneck, 2004). Deci e Ryan (1985), ancorados nos estudos de White (1975) e DeCharms (1984), propuseram a chamada Teoria da Autodeterminação a fim de analisar os comportamentos dos indivíduos conforme o estímulo. A mesma defende que todo ser humano é dotado de uma capacidade natural para obter o desenvolvimento saudável e a auto regulação, e que desde o nascimento as pessoas envolvem-se em 
atividades que lhes permitam a satisfação de três necessidades psicológicas básicas: a competência, a autonomia e o vínculo (Ryan \& Deci, 2000a).

Seminalmente, a motivação era discutida somente como um construto unitário que oscilava na variável intensidade (muito motivado ou pouco motivado) (Deci \& Ryan, 1985). No entanto, a partir da década de 90, as investigações empíricas aprimoraram a variável, explorando-a a partir da qualidade e tipos de motivação, ou ainda, os objetivos que levam o indivíduo a tomar determinada decisão. As discussões passam então a fazer distinção entre a motivação intrínseca e extrínseca (Deci \& Ryan, 1991; Ryan \& Deci, 2000a; 2000b). Posteriormente, Gagné e Deci (2005) avançam propondo uma linha contínua de autodeterminação a partir de três grupos: (i) a desmotivação, (ii) a motivação intrínseca e (iii) a motivação extrínseca.

A desmotivação compreende a inexistência de pro atividade e interesse por parte do indivíduo (Boruchovitch \& Bzuneck, 2004; Gagné \& Deci, 2005; Guimarães \& Bzuneck, 2008), a pessoa não apresenta intenção nem comportamento proativo, assim em tal situação, observa-se a falta de percepção de controle pessoal (Guimarães \& Bzuneck, 2008). Assim apresenta-se a primeira hipótese do estudo, propondo que: H1a: A desmotivação influencia negativamente a adoção da estratégia de estudo relativa a aquisição de conhecimento aprofundado; H1b: A desmotivação influencia positivamente a adoção da estratégia de estudo relativa a aquisição de conhecimento superficial.

A motivação intrínseca, por sua vez, envolve a ação do indivíduo estimulada pelo interesse individual, derivada da satisfação espontânea ou prazer pela atividade (Boruchovitch \& Bzuneck, 2004; Gagné \& Deci, 2005; Guimarães \& Bzuneck, 2008). Nela a pessoa tem interesse e prazer na realização da tarefa, sendo a atividade vista como um fim em si mesma (Guimarães \& Bzuneck, 2008). Assim, espera-se que essa motivação influencie na estratégia de estudo; fato que leva a propor que: H2a: A motivação intrínseca influencia positivamente a adoção da estratégia de estudo relativa a aquisição de conhecimento aprofundado; H2b: A motivação intrínseca influencia negativamente a adoção da estratégia de estudo relativa a aquisição de conhecimento superficial.

Na motivação extrínseca, por sua vez, a atividade é um instrumental para atingir eventos externos desejáveis, recompensas tangíveis, ou ainda, esquivar-se de situações indesejáveis. A satisfação, nesse caso, é consequência de contingências/regulações externas de sua conduta. Nessa categoria, os níveis de motivação externa são definidos como regulação introjetada, identificada e integrada (Boruchovitch \& Bzuneck, 2004; Gagné \& Deci, 2005; Guimarães \& Bzuneck, 2008).

Um regulamento aceito pela pessoa, mas que não condiz com seus princípios pessoais é denominado como introjetado. Nesse tipo de regulação, a condição é interna a pessoa, mas é relativamente controlada por meio de uma motivação extrínseca, levando-o a agir a fim de garantir sentimentos como dignidade, ego, autoestima e evitar a culpa ou vergonha (DeCharms, 1984). Dessa forma, a terceira hipótese formulada para o estudo é: H3a: A motivação introjetada influencia negativamente a adoção da estratégia de estudo relativa a aquisição de conhecimento aprofundado; H3b: A motivação introjetada influencia positivamente a adoção da estratégia de estudo relativa a aquisição de conhecimento superficial.

A regulação identificada é entendida como um tipo de motivação extrínseca internalizada, que ocorre quando o valor do comportamento é reconhecido como pessoalmente valioso. Nesse caso, os indivíduos sentem maior liberdade e vontade em envolver-se na atividade, uma vez que há a relevância pessoal e o comportamento reflete em um bem estar em si mesmo. Pesquisas empíricas que discutem a regulamentação identificada por vezes atrelam essa variável a motivação intrínseca para compreender a motivação autônoma (Vansteenkiste, Lens, De Witte, De Witte \& Deci, 2004b). A regulação identificada é mais autônoma do que as demais, visto que, já possui interiorização, mesmo que a razão para fazer alguma coisa seja de origem externa, essa motivação do indíviduo é instrumental (Guimarães \& Bzuneck, 2008). Assim espera-se que: H4a: A motivação identificada influencia positivamente a adoção da estratégia de estudo relativa a aquisição de conhecimento aprofundado; H4b: A motivação identificada influencia negativamente a adoção da estratégia de estudo relativa a aquisição de conhecimento superficial.

Por fim, na regulação integrada as pessoas têm o pleno senso de que o comportamento é parte integrante de quem eles são, que ele emana o senso de si mesmo e, portanto, é autodeterminado. Esse tipo de motivação representa a forma de motivação extrínseca mais avançada pois compartilha algumas qualidades da motivação intrínseca. Embora apresente a característica de autonomia, a regulamentação integrada ainda é considerada como externa, pois a motivação não surge internamente no interesse pessoal da atividade, mas pela significância que a atividade possui para atingir objetivos pessoais (Gagné \& Deci, 2005). Possui uma coerência entre o comportamento, os objetivos e valores do individuo, é uma motivação extrínseca mais autônoma, mesmo que o foco ainda pareia nos benefícios pessoais originados da realização da atividade (Guimarães \& Bzuneck, 2008). Assim, propõe-se que: H5a: A motivação integrada influencia negativamente a adoção da estratégia de estudo relativa a aquisição de conhecimento aprofundado; H5b: A motivação integrada influencia positivamente a adoção da estratégia de estudo relativa a aquisição de conhecimento superficial.

De modo geral, as regulações externas estimulam um comportamento guiado por contingências diante da promessa de uma recompensa ou ameaça de uma punição (Guimarães \& Bzuneck, 2008). Assim, acredita-se que existem diversos outros elementos que compõem estímulos externos à motivação (Guimarães \& Bzuneck, 2008). Portanto, propõe-se que: H6a: A regulação externa por frequência as aulas influencia negativamente a adoção da estratégia de estudo relativa a aquisição de conhecimento aprofundado; H6b: A regulação externa por frequência as aulas influencia positivamente a adoção da estratégia de estudo relativa a aquisição de conhecimento superficial; H7a: A regulação externa por recompensas sociais influencia negativamente a adoção da estratégia de estudo relativa a aquisição de conhecimento aprofundado; H7b: A regulação externa por recompensas sociais influencia positivamente a adoção da estratégia de estudo relativa a aquisição de conhecimento superficial. 
Na perspectiva educacional, os princípios dessa teoria são aplicados na observação de como manifestações de motivação podem afetar de diversas formas o processo de ensino-aprendizagem (Leal et al., 2013). Acredita-se que as interações no contexto social existentes em sala de aula poderiam atuar frustrando ou satisfazendo total ou parcialmente os anseios individuais, tornando a motivação autônoma um sentimento contingente (Ryan \& Deci, 2000a; 2000b; Reeve, Deci \& Ryan, 2004; Hagger, Chatzisarantis \& Harris, 2006).

Estudos empíricos relacionam também melhores resultados em termos de aprendizagem, desempenho e criatividade a partir da existência de motivação intrínseca no indivíduo. A motivação extrínseca, menos elaborada, comumente contraposta à motivação intrínseca, foi associada a efeitos mais reservados sobre os comportamentos (Ryan \& Deci, 2000a; 2000b; Vansteenkiste et al., 2004a; Kolb \& Kolb, 2005; Ferreira, 2009; Kusurkar et al., 2012; Carmo, 2014). Além desses aspectos, sabe-se que a qualidade da motivação de uma pessoa para realização de determinada tarefa também pode diferenciar-se entre níveis de autodeterminação (Deci \& Ryan 1991; Ryan \& Deci, 2000a).

Convém ressaltar que as motivações são individuais e orientadas por situações que dão subsídios a necessidades psicológicas, que consequentemente determinam os esforços a serem realizados (Sobral, 2003). Diante desse contexto, surge então o interesse em compreender os elementos que levam o aluno a querer fazer algo e a intensidade a ser aplicada para realizar tal tarefa, uma vez que acredita-se que o seu tipo motivação pode atuar na modalidade de estratégia adotada no estudo. Esta relação será discutida na próxima seção.

\subsection{Relações entre estratégias de aprendizagem e motivação dos alunos}

Alunos se deparam diariamente com diversos momentos de crescimento, tanto de natureza técnico-profissional, como de desenvolvimento individual enquanto ser humano e agente transformador. No ambiente educacional, as estratégias de ensino-aprendizagem são compreendidas como o caminho que os alunos adotam para alcançar objetivos fixados (Morin, 2004). As mesmas referem-se ao modo ou estilo como os alunos estudam um conteúdo, seja ele a fim de compreender sua essência ou somente decorá-lo (Biggs, Kember \& Leung, 2001). Kolb e Kolb (2005) relatam que, sendo o processo de aprendizado o feedback de experiências, espera-se que o ambiente educacional desenvolva a compreensão não apenas do "como fazer", mas também do "por que fazer". Assim, os alunos não estariam limitados a decorar as respostas ou gravar o assunto somente com a finalidade de ser aprovado nas avaliações (conhecimento superficial/ instrumental), mas entender os objetivos reais de um conteúdo ou de compreender seus princípios subjacentes (conhecimento aprofundado) (Wanous, Procter \& Murshid, 2009; Munshi et al., 2012).

Respaldado na Teoria da Autodeterminação, Deci e Ryan (1985) defendem que um comportamento intrinsecamente motivado é o protótipo de atividades autodeterminadas, uma vez que os interesses dos indivíduos são motivados por uma cadeia autossustentável. Quando intrinsecamente estimuladas, as pessoas se envolvem em uma tarefa com a finalidade de compreensão da causa, e não somente com objetivo de obter um resultado específico. Nesse caso, o aluno molda-se a fim de maximizar o significado no conteúdo apresentado (Biggs et al., 2001). Além disso, a motivação, quando de origem autônoma ou voluntária, atua significativamente em um melhor aprendizado e maior bem-estar (Ryan \& Deci, 2000a). Em contrapartida, comportamentos extrinsecamente motivados são realizados a fim de atingir um fim instrumental (Ryan \& Deci, 2000a), e o discente assimila o assunto para fins de memorização de fatos (Biggs et al., 2001).

No Brasil, a Teoria da Autodeterminação é aplicada na análise de diferentes variáveis, dentre elas, nos níveis de motivação entre disciplinas (Guimarães et al., 2002), nos diferentes períodos de um curso (Oliveira et al., 2010), no mapeamento de diferentes motivações no curso (Leal, Miranda \& Carmo, 2013), como também entre instituições com diferentes modelos de gestão (Lopes et al., 2015). Pesquisas internacionais avançam utilizando a teoria para documentar relações entre a motivação e o nível estratégia de aprendizado (mais aprofundada ou superficial/instrumental) (Vansteenkiste et al., 2004a; Vansteenkiste et al., 2005; Kusurkar et al., 2012; Munshi, et al., 2012).

Guimarães et al. (2002) mostraram que existem diferenças significativas em relação as variáveis sociais demográficas, como sexo e o tipo de motivação. Oliveira et al. (2010), não conseguiram diagnosticar um momento de transição entre níveis de motivação extrínseca e motivação intrínseca em função do grau de maturidade dos acadêmicos de Ciências Contábeis. Leal, Miranda e Carmo (2013) observaram que alunos dos últimos anos do curso apresentaram maiores índices das modalidades de motivação introjetada, externa e de desmotivação. Já Lopes et al. (2015), ao investigar divergências nos níveis de motivação entre os alunos de universidades públicas e privadas, não observaram diferenças na análise da categoria.

Internacionalmente, Vansteenkiste et al. (2004a) analisaram as relações entre os objetivos intrínsecos e extrínsecos, os níveis de aprendizagem autônoma e controlada, o desempenho e a persistência nos estudos. Os achados confirmaram que as motivações produziram efeitos na profundidade de processamento da aprendizagem, no desempenho e na persistência. Vansteenkiste et al. (2005) mostraram que a motivação autônoma influencia positivamente diversas variáveis, dentre elas, as estratégias de aprendizagem, o sucesso acadêmico, o processamento da informação, a concentração, o gerenciamento do tempo e o bem-estar dos estudantes. Por outro lado, as motivações de ordem controlada foram associadas a maiores taxas de abandono escolar, menores atitudes de aprendizagem adaptativa e a indisposição ao estudo.

Munshi et al. (2012) mostrou que alunos orientados por uma estratégia de aprendizado instrumental possuem motivações externas, enquanto aqueles que optam por um conhecimento aprofundado apresentam motivações internas. Kusurkar et al. (2012) encontraram que a motivação autônoma afeta positivamente na dedicação e na adoção de uma boa estratégia de estudo. Além disso, foi observado também um efeito positivo das variáveis no desempenho acadêmico. 
A pesquisa adiciona a esse contexto resultados significativos na análise das motivações para grupo de homens (maior motivação controlada) e de mulheres (maior motivação autônoma). A partir dessas considerações, entende-se que o presente estudo, ao propor a análise dessas relações em cenário nacional, pode proporcionar significativas contribuições para compreensão do ambiente de aprendizagem. A próxima seção destina-se a esclarecer os procedimentos aplicados para atingir o objetivo de verificar os efeitos existentes entre as variáveis apresentadas.

\section{PROCEDIMENTOS METODOLÓGICOS}

O estudo adota uma abordagem descritiva de caráter quantitativo por meio de uma survey. A população compreendeu 135 alunos do curso de Ciências Contábeis de uma universidade pública do Paraná. A seleção da amostra ocorreu por acessibilidade (um câmpus da universidade). Os dados foram coletados por meio de um questionário aplicado presencialmente em sala de aula, mediante agendamento e autorização do coordenador do curso.

O instrumento de pesquisa foi estruturado em três blocos: (i) dados demográficos, (ii) motivação, e, (iii) estratégia de estudo. A escala empregada foi do tipo likert. O construto motivação composto por 29 assertivas, foi adaptado do estudo de Guimarães e Bzuneck (2008). Já as estratégias de estudo, foram operacionalizadas a partir de Biggs et al. (2001), Kusurkar et al. (2012) e Genc e Tinmaz (2013), perfazendo 20 assertivas. A coleta foi realizada no mês de dezembro de 2017. Foram obtidas 106 respostas válidas, correspondente a 78\% da população estudada. Na Figura 1, apresentam-se os construtos que nortearam a pesquisa.

Figura 1.Constructos da pesquisa

\begin{tabular}{|c|l|c|}
\hline Construto & Variáveis & Fonte \\
& Desmotivação & \\
Regulação integrada & Regulação externa por frequência às aulas \\
Motivação & Regulação introjetada & Guimarães e Bzuneck (2008) \\
& Regulação externa por recompensas sociais & \\
Regulação Identificada & Motivação Intrínseca \\
Estratégia de estudo & Conhecimento compreensível/aprofundado & \\
& Conhecimento instrumental/superficial & Biggs et al. (2001), Kusurkar et al. (2012), \\
\end{tabular}

Fonte: elaborado pelos autores

Na Figura 1 mostra-se que o estudo fundamenta-se em dois construtos. A motivação respaldada na Teoria da Autodeterminação e subdivide-se em sete elementos: desmotivação, regulação integrada, regulação externa por frequência às aulas, regulação introjetada, regulação externa por recompensas sociais, regulação identificada e motivação intrínseca, conforme abordado em Guimarães e Bzuneck (2008). O construto estratégia de aprendizagem, por sua vez, divide-se em dois itens: conhecimento compreensível ou aprofundado e conhecimento instrumental ou superficial. O instrumento procede do Questionário do Processo de Estudo (R-SPQ-2F), desenvolvido em Biggs et al. (2001) e posteriormente abordado em Kusurkar et al. (2012) e Genc e Tinmaz (2013), ao qual tem por finalidade verificar se os alunos compreendem os problemas subjacentes ao conteúdo ou se preferem reconhecer somente os pontos-chave.

Diante do objetivo de verificar se a motivação dos alunos influencia nas estratégias de estudo adotadas na aprendizagem acadêmica, foram testadas as sete hipóteses formuladas e segmentadas em "a" e "b" (ao total, catorze). Para a análise dos dados e teste das hipóteses empregou-se a técnica de análise fatorial de modo a reduzir as variáveis em fatores e, com os dados da análise fatorial, realizou-se a estatística descritiva. O teste de Alpha de Cronbach foi analisado a fim de verificar a confiabilidade dos fatores. Para o teste de hipóteses e análise das relações entre a motivação do acadêmicos e as estratégias de aprendizagem, empregou-se a técnica de regressão múltipla por meio do software SPSS. Segundo Hair Jr. et al. (2009), esse tipo de análise permite avaliar o comportamento de uma variável dependente a partir do comportamento de uma ou mais variáveis independentes. Além disso, dois modelos foram testados nesse estudo, um deles aplicando como variável dependente o fator relativo as estratégias de conhecimento aprofundado, e o outro a partir das estratégias de conhecimento superficial. Sendo assim, as variáveis são categorizadas conforme Figura 2. 
Figura 2. Enquadramento das variáveis nos modelos de regressão

\begin{tabular}{|l|l|l|}
\hline Variável/fator & Comportamento da variável & Modelo de enquadramento \\
Desmotivação & & \\
Regulação Integrada & Variável independente & Modelo 1 e 2 \\
$\begin{array}{l}\text { Regulação Introjetada } \\
\text { Regulação Externa por recompensas sociais } \\
\text { Regulação Identificada }\end{array}$ & \\
$\begin{array}{l}\text { Motivação Intrínseca } \\
\text { Conhecimento compreensível/aprofundado }\end{array}$ & Variável dependente & \\
Conhecimento instrumental/superficial & & Modelo 1 \\
\hline
\end{tabular}

Fonte: elaborado pelos autores

A validação dos modelos de regressão contou com a verificação dos testes: i) White para verificar a homocedasticidade dos dados, ii) fator de inflação de variância (VIF) para verificação de presença de multicolineariedade entre as variáveis independentes, iii) Durbin Watson para descartar a hipótese de presença de correlação entre os resíduos, iv) RESET de Ramsey para identificar possíveis erros de especificação na forma funcional de um modelo de regressão, e v) coeficiente de determinação $\left(\mathrm{R}^{2}\right)$ para avaliar o poder explicativo da modelagem (Hair Jr. et al., 2009).

\section{ANÁLISE E DISCUSSÃO DOS RESULTADOS}

A apresentação dos resultados desse estudo inicia-se com ao perfil dos respondentes da pesquisa. Na Tabela 1, dispõe-se os dados demográficos dos participantes do estudo.

Tabela 1. Perfil dos respondentes

\begin{tabular}{|c|c|c|c|c|c|}
\hline Gênero & Quantid. & Percentual & Idade & Quantid. & Percentual \\
\hline Masculino & 40 & $37,73 \%$ & 17 & 2 & $1,88 \%$ \\
\hline Feminino & 66 & $62,27 \%$ & 18 & 17 & $16,05 \%$ \\
\hline Total & 106 & $100 \%$ & 19 & 18 & $17 \%$ \\
\hline Período & Quantid. & Percentual & 20 & 20 & $18,86 \%$ \\
\hline 1 & 37 & $34,90 \%$ & 21 & 16 & $15,11 \%$ \\
\hline 2 & 27 & $25,47 \%$ & 22 & 11 & $10,37 \%$ \\
\hline 3 & 29 & $27,35 \%$ & 23 & 11 & $10,37 \%$ \\
\hline 4 & 13 & $12,28 \%$ & 24 & 6 & $5,66 \%$ \\
\hline \multirow[t]{4}{*}{ Total } & 106 & $100 \%$ & 26 & 2 & $1,88 \%$ \\
\hline & & & 28 & 2 & $1,88 \%$ \\
\hline & & & 39 & 1 & $0,94 \%$ \\
\hline & & & Total & 106 & $100 \%$ \\
\hline
\end{tabular}

Conforme Tabela 1, 62,27\% dos respondentes correspondem ao sexo feminino, enquanto $37,73 \%$ são do sexo masculino. Nota-se também que os períodos com maior concentração de respondentes foram os anos iniciais, isto porque a instituição adota uma modalidade de retenção na oferta de disciplinas para alunos reprovados. Por exemplo, se este é reprovado em uma das disciplinas do primeiro ano, o mesmo fica retido neste período e sua carga horária é adaptada para cursar prioritariamente a disciplina em dependência, e nos dias vagos/restantes, desde que não haja conflito de 
horário, as demais. Desse modo, os alunos matriculados no primeiro ano do curso representam 34,90\% da amostra, seguidos do terceiro período com $27,35 \%$, segundo período com $25,47 \%$ e último período, $12,28 \%$. Em relação a idade dos respondentes, $87,76 \%$ deles tem sua idade concentrada entre 18 e 23 anos.

Após esta etapa, realizou-se a análise fatorial exploratória (AFE) pelo método de Análise de Componentes Principais e rotacionada pela Varimax, de modo a reduzir e agrupar os itens do construto motivação em fatores comuns a partir dos 29 itens da escala de Guimarães e Bzuneck (2008). De acordo com Fávero, Belfiore, Silva e Chan (2009), recomenda-se, como regra geral, que as pesquisas empreguem um número de observações (amostra) pelo menos cinco vezes maior que o número de variáveis investigadas. Nesse sentido, os 106 participantes dessa pesquisa atendem a esse pré-requisito para aplicação da AFE, uma vez que as observações são cinco vezes maior que as 21 variáveis (itens) levantadas no instrumento aplicado no estudo. Os resultados dos primeiros testes, apontaram que os itens Q3, Q5, Q6, Q7, Q10, Q11, Q18 e Q24 não atingiram carga fatorial mínima recomendada (0,50), sendo então, excluídos do agrupamento (Hair Jr. et al., 2009). Após esse procedimento, a AFE agrupou 7 fatores por meio da combinação dos 21 itens remanescentes.

O resultado obtido no teste de Kaiser-Meyer-Olkin (KMO), com valor de 0,720, mostrou adequação conceitual média da amostra à analise fatorial a partir da análise das inter correlações entre as variáveis. Neste indicador a obtenção de valores inferiores a 0,600 sugerem que a análise fatorial não é satisfatória, haja vista a existência de uma correlação fraca entre as variáveis (Fávero et al., 2009). O teste de esfericidade Bartlett indicou também significância geral das correlações, cujo valor obtido foi de 12,246, df.=15 e p=0,000 (Hair Jr. et al., 2009). Uma vez confirmada a adequação dos agrupamentos realizados, na Tabela 2 apresenta-se os fatores apurados para os itens do questionário.

Tabela 2. Análise fatorial exploratória (Varimax)

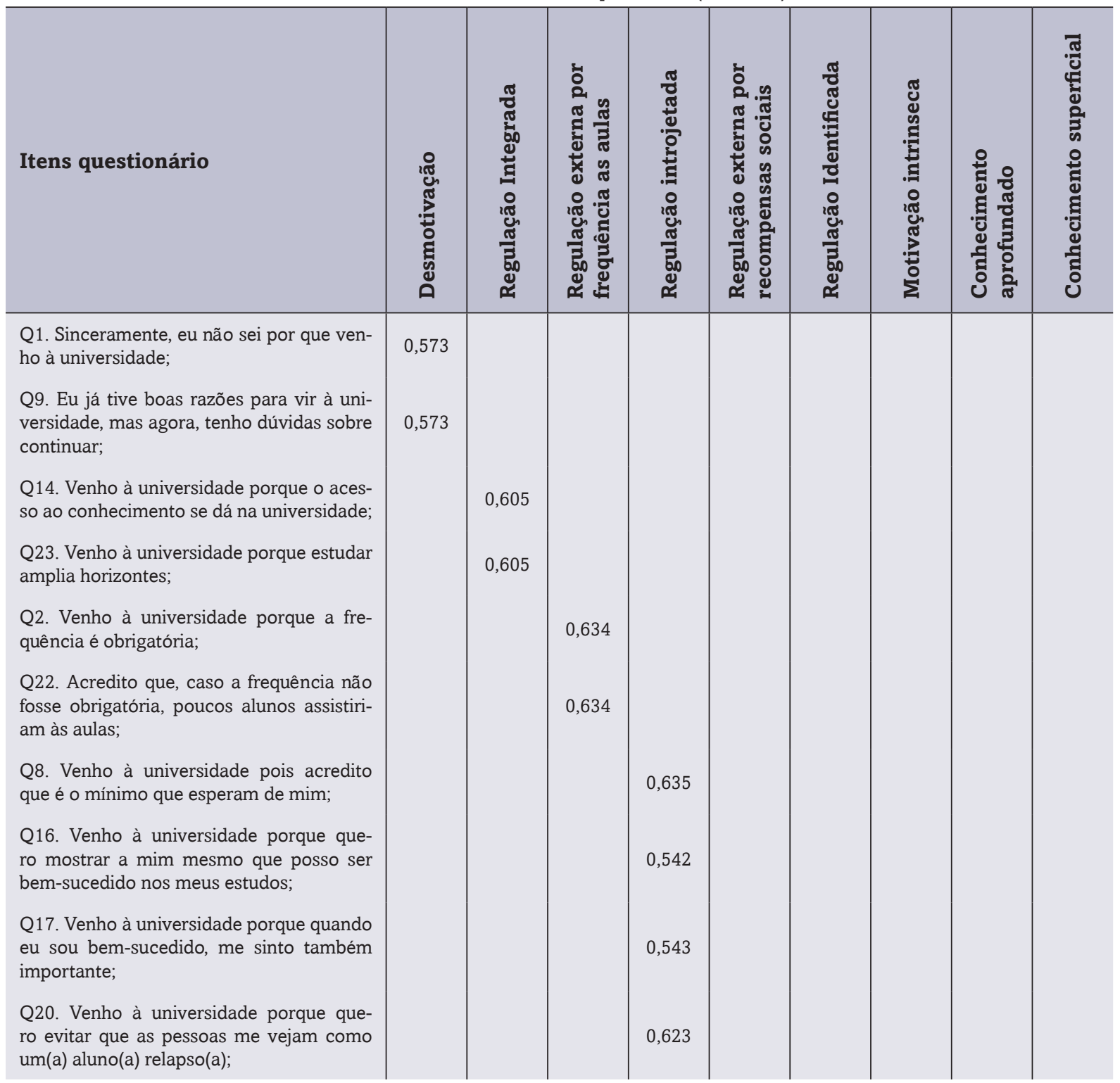


Itens questionário

Q25. Venho à universidade porque enquanto estiver estudando não preciso trabalhar;

Q26. Ver meus amigos é o principal motivo pelo qual venho à universidade;

Q19. Venho à universidade porque acredito que a cobrança de presença é necessária para que os alunos levem o curso a sério e venham para as aulas;

Q21. Venho à universidade porque a frequência nas aulas é necessária para a aprendizagem;

Q4. Venho à universidade pelo prazer que tenho em me envolver em debates/conversas com professores interessantes;

Q13. Venho à universidade porque para mim a universidade é um prazer;

Q1. Eu acho que estudar me dá um sentimento de profunda satisfação pessoal;

Q2. Eu preciso ter muita dedicação em uma disciplina para que eu possa formar minhas próprias conclusões antes de eu estar satisfeito;

Q6. Como acho a maioria dos novos tópicos interessantes, muitas vezes, gasto um tempo extra tentando aprender mais sobre o conteúdo/informações sobre eles;

Q9. Eu acho que estudar tópicos acadêmicos pode ser, às vezes, tão interessante quanto um bom romance ou filme.

Q10. Foco em tópicos/disciplinas importantes até que eu os compreenda completamente;

Q13. Eu me esforço em meus estudos porque eu acho o conteúdo interessante;

Q14. Passo muito do meu tempo buscando mais tópicos adicionais aos foram discutidos em aulas;

Q18. Faço questão de ver as leituras sugeridas nas aulas;

Q3. Meu objetivo é passar no curso fazendo o menor trabalho possível;

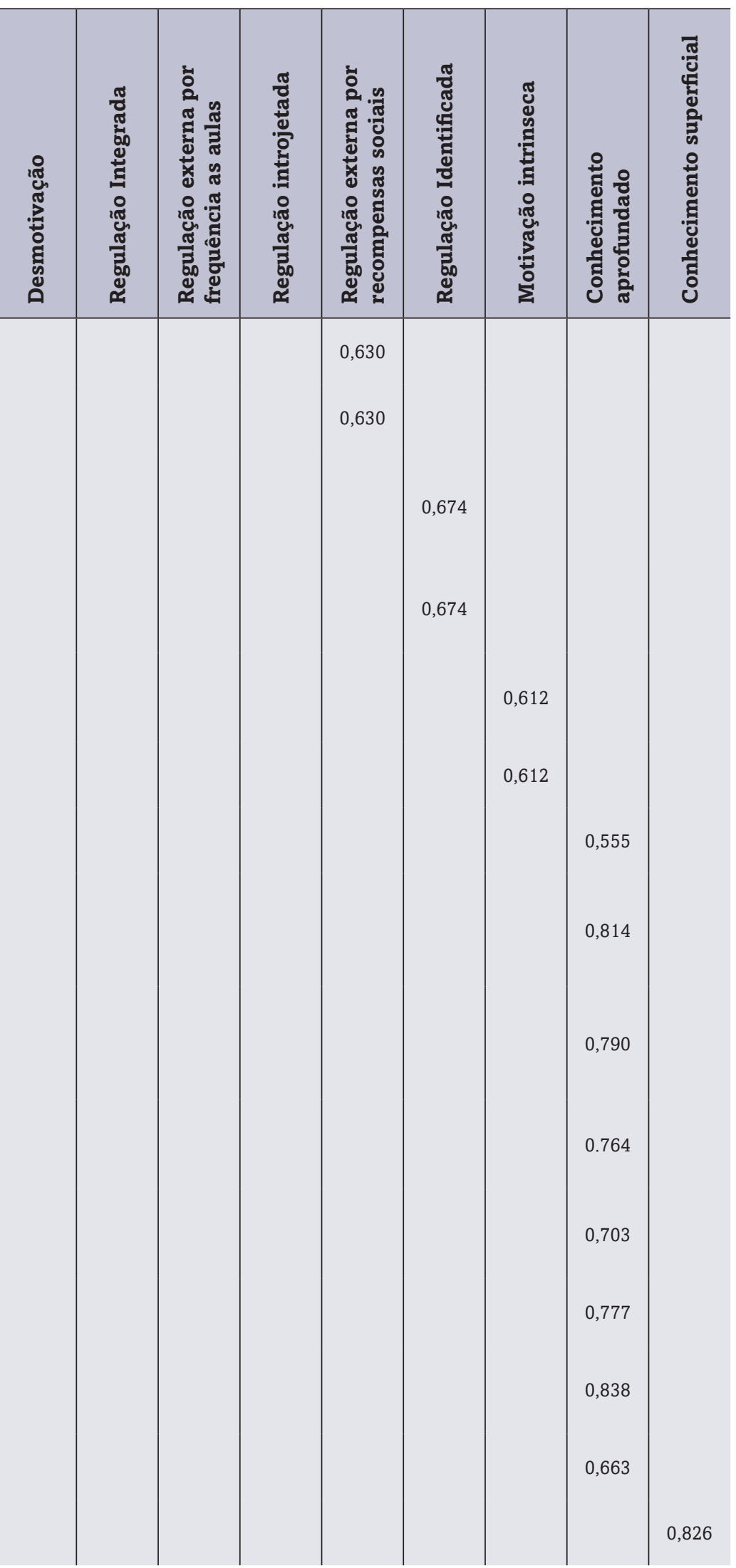






Fonte: dados da pesquisa

Na Tabela 2 apresenta-se a formação de 9 fatores a partir do instrumento de pesquisa. Eles são divididos em dois blocos, o primeiro deles de motivação (7 fatores) e o segundo para estratégia de pesquisa (2 fatores). O Bloco 1 contou com 21 itens que foram reduzidos em 7 fatores, os quais explicaram $61,55 \%$ da variação total dos dados. Todos os itens obtiveram cargas fatoriais aceitáveis, superiores a 0,50 (Hair Jr. et al., 2009). Após a aplicação da análise fatorial exploratória, calculou-se a confiabilidade do questionário aplicado na pesquisa por meio do Alpha de Cronbach. Embora as escalas já tenham sido validadas por outros estudos, os dados mostraram que os alfas para os construtos (1) regulação externa por frequência as aulas, (2) externa por recompensas sociais e (3) regulação identificada não atingiram o mínimo aceitável de 0,60. Os demais fatores demonstraram-se bons para uma pesquisa exploratória (Hair Jr. et al., 2009).

Os resultados expostos na Tabela 2 mostram que o fator 1, relativo a desmotivação existente nos alunos, agrupou os itens Q1 e Q9 do instrumento de pesquisa. O fator 2, responsável pela regulação integrada, agregou os itens Q14 e Q23. O fator 3, representado pela regulação externa por frequência nas aulas, formou-se pelos itens Q2 e Q22. O fator 4, sobre a regulação introjetada, compôs-se de Q8, Q16, Q17 e Q20. Já o fator 5, sobre a regulação externa por recompensas sociais, associou os itens Q25 e Q26. O fator 6, regulação identificada, reuniu Q19 e Q21. Já o fator 7, relacionado a motivação intrínseca, formou-se pelos itens Q4 e Q13. Os achados comportaram-se divergentes de estudos que adotaram tal escala, como de Leal, Miranda e Carmo (2003), Guimarães e Bzuneck (2008) e Souza (2008). Acredita-se que essa diferença pode ter sido ocasionada por alguns fatores, dentre eles, variável temporal, cultura regional, tipo de amostra e idade. 
No Bloco 2, os 20 itens relacionados a estratégia de estudo foram reduzidos a 2 fatores, os quais explicaram $63,30 \%$ da variação total dos dados. Todos os itens obtiveram cargas fatoriais e Alpha de Cronbach aceitáveis - maiores que 0,50 e 0,60 (Hair et al., 2009). Na análise fatorial exploratória da estratégia, considerou-se os 20 itens da escala resultante da pesquisa de Biggs et al. (2001), Kusurkar et al. (2012) e Genc e Tinmaz (2013). No entanto, após os primeiros testes, os itens Q5, Q16, Q17, e Q20 foram excluídos por não apresentarem carga fatorial mínima recomendada. Assim, a análise fatorial resultou em 2 fatores, por meio da combinação dos 16 itens remanescentes (Tabela 2). Após realizados os testes de análise fatorial e verificados os indicadores de validade interna do instrumento, realizou-se o teste de estatística descritiva dos fatores, conforme Tabela 3.

Tabela 3. Estatística descritiva por fator

\begin{tabular}{|c|c|c|}
\hline Fatores & Média & s \\
\hline Desmotivação & 1,585 & 1,0856 \\
\hline Regulação Integrada & 5,377 & 1,4829 \\
\hline Regulação Externa por frequência às aulas & 2,208 & 1,5226 \\
\hline Regulação Introjetada & 4,736 & 1,8011 \\
\hline Regulação Externa por recompensas sociais & 1,802 & 1,4304 \\
\hline Regulação Identificada & 3,000 & 1,7403 \\
\hline Motivação Intrínseca & 3,915 & 1,3878 \\
\hline Conhecimento aprofundado & 3,847 & 1,5882 \\
\hline Conhecimento superficial & 2,888 & 1,6867 \\
\hline
\end{tabular}

Nota: $\mathrm{s}=$ Desvio padrão

Fonte: dados da pesquisa

Em relação a dispersão dos dados em torno da média amostral, observou-se que a desmotivação apresentou o menor coeficiente de desvio padrão $(s=1,0856)$ e uma média de 1,585 (média=1,585), indicando uma baixa variabilidade dos dados e um baixo nível de autodeterminação. Os índices encontrados para as motivações por regulação externa por recompensas sociais $(s=1,4304$; média $=1,802)$ e frequência as aulas $(s=1,5226$; média $=2,208)$ também mostram que os alunos parecem pouco influenciados por esses estímulos externos. A variável frequência as aulas apurou a terceira menor média (média=2,208), o que parece mostrar que os alunos não vão à universidade somente pela presença, como também pelo aprendizado.

Alguns fatores apresentaram maiores distanciamentos dos coeficientes em relação à média, como é o caso das regulações introjetada ( $s=1,8011)$ e identificada ( $s=1,70403)$. Já, a motivação introjetada, aquela em que o aluno é movido por pressões internas, como culpa e ansiedade, apresentou média de 4,736, a segunda maior encontrada. Os resultados divergem dos estudos de Leal, Miranda e Carmo (2013) e Guimarães e Bzuneck (2008), os quais apresentaram médias inferiores a esta. Quanto à motivação intrínseca, onde s=1,3878 e média=3,915, observou-se um nível significativo de estímulo interno de modo que parte significativa dos discentes realizam atividades acadêmicas de forma espontânea, ou seja, para sua própria satisfação. O resultado assemelha-se ao de Guimarães e Bzuneck (2008).

Em relação as estratégias de estudos, o fator que representa a utilização de técnicas de aprendizagem para conhecimento superficial mostrou-se com dados mais dispersos em relação aos demais $(s=1,6867$; média=2,888), ou seja, o comportamento dos respondentes comportou-se com maior variação em relação a média apresentada. Quanto as estratégias de conhecimento aprofundado, a média (média=3,847) mostra uma propensão positiva dos discentes a compreender a causa e utilidade dos conteúdos vivenciados em sala de aula.

Após a análise fatorial exploratória e a estatística descritiva, realizou-se testes de validação dos modelos de regressão; o primeiro deles com a variável explicada conhecimento aprofundado (modelo 1) e o segundo com a de conhecimento superficial (modelo 2). Identificou-se a homocedasticidade nos dados por meio do teste de White, no qual apontou para o modelo $1 \mathrm{LM}=0,81$ e $p$-valor=0,3695, e para o modelo $2 \mathrm{LM}=0,91$ e $p$-valor=0,3392. Observou-se baixa multicolinearidade entre as variáveis independentes, conforme observado na Tabela 4, onde os VIF possuem valores inferiores a 5 (Hair Jr. et al., 2009).

A adequação do modelo foi diagnosticada pelo teste RESET, obtendo-se $\mathrm{F}=1,93$ e $p$-valor=0,1229 no modelo 1 , e F=2,42 e $p$-valor=0,0709 no modelo 2. O teste Durbin-Watson (DW=1,953) descartou a presença de autocorrelação residual (1<estatística de Durbin-Watson<3) (Hair Jr. et al., 2009). Em seguida, a análise de regressão múltipla foi realizada pelo método de estimação STEPWISE, a fim de verificar a existência de relações entre as variáveis relativas a fatores motivacionais e as estratégias de ensino (oriundos da AFE). Os resultados da regressão múltipla são demonstrados na Tabela 4. 
Tabela 4. Coeficientes da Regressão

\begin{tabular}{|c|c|c|c|c|c|c|}
\hline \multicolumn{7}{|c|}{ Modelo 1 (variável explicada: conhecimento aprofundado) } \\
\hline & Coef. b & VIF & Erro padrão & Razão t & P-valor & Hipótese \\
\hline Constante & $-0,172$ & - & 0,460 & 0,375 & 0,709 & - \\
\hline F1: Desmotivação & $-0,289$ & 1,684 & 0,080 & $-3,627$ & $0,000 *$ & H1a \\
\hline F2: Regulação integrada & $-0,190$ & 1,773 & 0,079 & $-2,388$ & $0,019 * \star$ & $\mathrm{H} 5 \mathrm{a}$ \\
\hline $\begin{array}{l}\text { F3: Regulação externa por } \\
\text { frequência às aulas }\end{array}$ & 1,150 & 1,000 & 0,130 & 8,850 & $0,000^{*}$ & $\mathrm{H} 6 \mathrm{a}$ \\
\hline F4: Regulação introjetada & $-0,122$ & 1,222 & 0,057 & $-2,155$ & $0,034^{\star \star}$ & H3a \\
\hline $\begin{array}{l}\text { F5: Regulação externa por } \\
\text { recompensas sociais }\end{array}$ & $-0,156$ & 1,311 & 0,072 & $-2,168$ & $0,033^{\star \star}$ & $\mathrm{H} 7 \mathrm{a}$ \\
\hline F6: Regulação identificada & $-0,270$ & 1,329 & 0,084 & $-3,216$ & $0,002^{*}$ & $\mathrm{H} 4 \mathrm{a}$ \\
\hline F7: Motivação intrínseca & 0,256 & 1,226 & 0,058 & 4,396 & $0,000 *$ & $\mathrm{H} 2 \mathrm{a}$ \\
\hline $\mathrm{F}$ & 19,89 & & & & & \\
\hline P-Valor (F) & 0,000 & & & & & \\
\hline $\mathrm{R}^{2}$ Ajustado & 0,639 & & & & & \\
\hline \multicolumn{7}{|c|}{ Modelo 2 (variável explicada: conhecimento superficial) } \\
\hline & Coef. b & VIF & Erro padrão & Razão t & P-valor & Hipótese \\
\hline Constante & 1,327 & - & 0,304 & 4,365 & $0,000^{*}$ & - \\
\hline F1: Desmotivação & $-0,319$ & 1,271 & 0,090 & $-3,566$ & $0,001^{*}$ & $\mathrm{H} 1 \mathrm{~b}$ \\
\hline F2: Regulação integrada & $-0,650$ & 2,429 & 0,094 & $-6,934$ & $0,000^{*}$ & $\mathrm{H} 5 \mathrm{~b}$ \\
\hline $\begin{array}{l}\text { F3: Regulação externa por } \\
\text { frequência às aulas }\end{array}$ & 0,529 & 1,167 & 0,163 & 3,245 & $0,002^{\star}$ & $\mathrm{H} 6 \mathrm{~b}$ \\
\hline F4: Regulação introjetada & 0,122 & 1,222 & 0,057 & 2,155 & 0,034 ** & $\mathrm{H} 3 \mathrm{~b}$ \\
\hline $\begin{array}{l}\text { F5: Regulação externa por } \\
\text { recompensas sociais }\end{array}$ & 2,166 & 3,709 & 0,205 & 10,589 & $0,000 *$ & $\mathrm{H} 7 \mathrm{~b}$ \\
\hline F6: Regulação identificada & 0,576 & 1,000 & 0,109 & 5,298 & $0,000 *$ & $\mathrm{H} 4 \mathrm{~b}$ \\
\hline F7: Motivação intrínseca & $-0,274$ & 1,350 & 0,054 & $-5,065$ & $0,000^{*}$ & $\mathrm{H} 2 \mathrm{~b}$ \\
\hline $\mathrm{F}$ & 11,56 & & & & & \\
\hline P-Valor (F) & 0,000 & & & & & \\
\hline $\mathrm{R}^{2}$ Ajustado & 0,661 & & & & & \\
\hline
\end{tabular}

Notas: ${ }^{*}$ sig. $1 \%{ }^{* \star}$ sig. $5 \%$ *** sig. $10 \%$

Coef. b. = coeficiente beta

Fonte: dados da pesquisa

Os resultados da regressão múltipla mostram que os modelos propostos possuem um poder de explicação de $R^{2}$ $=0,639$ (modelo 1) e $R^{2}=0,661$ (modelo 2), ou seja, que respectivamente $63,90 \%$ e $66,10 \%$ das variáveis designadas dependentes (tipo de estratégia de aprendizagem) podem ser explicadas pelos regressores presentes no modelo (tipo de motivação). Os fatores motivacionais apresentaram correlação significante com as estratégias de estudo dos alunos. Após validado o modelo, testou-se as hipóteses. Ressalta-se que o gênero foi testado adicionalmente como variável de controle, mas não obteve significância no estudo em relação ao efeito da motivação sobre as estratégias de aprendizagem adotadas pelos alunos.

A H1a assume que a desmotivação dos acadêmicos é capaz de associar-se de forma negativa a busca por conhecimento aprofundado pelo aluno, enquanto $\mathrm{H} 1 \mathrm{~b}$ testa a relação positiva dessa variável sobre as estratégias de conhecimento superficial. A capacidade de predição das variáveis hipotetizadas foram confirmadas ( $p$-valor=0,000 e $p$-valor=0,001), 
e como esperado em H1a, verificou-se que as estratégias de conhecimento aprofundado são capazes de associar-se negativamente ao nível de desmotivação dos acadêmicos ( $b=-0,289$-valor $<0,001)$. Por outro lado, o coeficiente H1b indica uma associação negativa da variável desmotivação para o modelo de conhecimento superficial, fato que impossibilita aceitar a hipótese alternativa $(b=-0,319 p$-valor $<0,05)$. Nesse sentido, observou-se que a desmotivação diminui a opção pelo emprego de adoção de estratégias para conhecimento superficial. A H2a propõe que a motivação intrínseca do discente está associada de forma positiva ao emprego de estratégias aprofundadas para aquisição de conhecimento. Já, H2b defende que a motivação intrínseca relaciona-se negativamente ao uso de estratégias em busca de conhecimento superficial. Os resultados confirmam signigicativamente a previsão para as variáveis no modelo (ambas com p-valor $=0,000$ ). O coeficiente de $\mathrm{H} 2 \mathrm{a}$ mostra que a busca pelo conhecimento aprofundado parece aumentar a medida que os acadêmicos encontram-se mais motivados internamente $(\mathrm{b}=0,256 p$-valor $<0,001)$. Acredita-se também que o prazer intrínseco ao estudar tem como consequência maior desempenho acadêmico (Boruchovitch \& Bzuneck, 2004). Os resultados para $\mathrm{H} 2 \mathrm{~b}$ permitem inferir a relação negativa da motivação intrínseca sobre a estratégia de estudo superficial $(\mathrm{b}=0,274$ p-valor $<0,001)$, mostrando que alunos com protótipo de atividades autodeterminadas são avesos a aprendizagem por memorização. Em ambos casos, os achados não permitem rejeitar a hipótese alternativa.

A H3a assume que a motivação introjetada é capaz de predizer, a partir de uma associação negativa, a adoção de estratégias de estudo para conhecimento aprofundado. Já H3b propõe a relação positiva dessa variável na aquisição de conhecimento superficial. Os resultados apontam resultados estatisticamente significativos para as hipóteses ( $p$-valor=0,034 ambos) e permitem inferir que a motivação introjetada associa-se de forma negativa com o conhecimento aprofundado ( $b=-0,122$-valor $<0,05)$, e positiva com o conhecimento superficial $(b=0,122$-valor $<0,05)$. Desse modo, as análises observadas não permitem rejeitar H3a e H3b.

A H4a propõe a capacidade que a motivação identificada tem de predizer positivamente o emprego de estratégias de estudo aprofundando pelos acadêmicos; enquanto H4b defende que a motivação identificada relaciona-se negativamente com a opção por estratégias de estudo instrumentais. Os coeficientes encontrados para $\mathrm{H} 4 \mathrm{a}(\mathrm{b}=-0,270 p$-valor $<0,05)$ e H4b $(\mathrm{b}=0,576$-valor $<0,001)$ foram estatisticamente significativos. Os resultados mostram que a motivação identificada relaciona-se de forma negativa com a aprendizagem voltada ao conhecimento aprofundado, ou seja, mesmo aqueles indivíduos que, decorrente de elementos externos, reconhecem aquela ação como pessoalmente valiosa (motivação identificada) tendem a afastar-se do uso estratégias de compreensão de seus princípios subjacentes. Os resultados do modelo 2, indicam que a variável independente associa-se positivamente com as estratégias por conhecimento superficial, ou seja, o oposto a H4a é verdadeiro. Embora com efeitos significativos, os achados mostraram indicadores inversos às hipóteses formuladas, fato que permite rejeitar $\mathrm{H} 4 \mathrm{a}$ e $\mathrm{H} 4 \mathrm{~b}$.

A H5 foi segregada em H5a, onde testa a relação negativa da variável independente regulação integrada sobre o conhecimento aprofundado, e H5b, onde verifica a sua associação positiva sobre o conhecimento superficial. Os resultados confirmaram a significância estatística das hipóteses a H5a e H5b, indicando que a regulação integrada é capaz de predizer o emprego de estratégias de ensino para conhecimento instrumental ( $p$-valor=0,019 e $p$-valor=0,000). Ambos testes apontaram uma relação negativa com a forma de aprendizagem, sendo em H5a na proporção de 19\% (b=-0,190 p-valor $<0,05)$, e para H5b em 65\% ( $\mathrm{b}=-0,650$ p-valor $<0,001)$. Os achados apontados permitem que H5a não seja rejeitada e H5b seja rejeitada.

As hipóteses H6a e H6b foram definidas sobre a proposta de que a regulação externa por frequência às aulas relaciona-se negativamente com a estratégia de estudo aprofundada e positivamente as metodologias superficiais de assimilação de conteúdo. Conforme os resultados, os coeficientes de H6a e H6b foram estatisticamente positivos e significativos. Os modelos testados permitem inferir que a frequência às aulas relaciona-se positivamente, e mais fortemente, com o envolvimento aprofundado com as tarefas propostas $(\mathrm{b}=1,150$-valor $<0,001)$, mas a relação é também positiva com a estratégia de aprendizagem instrumental $(\mathrm{b}=0,529$-valor $<0,05)$. Dessa forma, os resultados permitem que H6a seja rejeitada, mas que $\mathrm{H} 6 \mathrm{~b}$ não seja.

A H7a assume a relação negativa da variável regulação externa por recompensas sociais sobre a forma de aquisição de conhecimento aprofundado, enquanto H7b testa sua associação positiva com o uso de metodologias de aquisição de conhecimento instrumental. Os resultados foram significativos para ambas hipóteses ( $p$-valor $\mathrm{H} 7 \mathrm{a}=0,033$ e H7b=0,000). Quanto aos coeficientes encontrados, $\mathrm{H7a}(\mathrm{b}=-0,156$-valor $<0,05)$ mostrou que as motivação derivadas de recompensas sociais se associam negativamente em $15,60 \%$ com o uso de metodologias que envolvam a compreensão intrínseca do assunto. Já o coeficiente de 2,166 para H7b (b=2,166 p-valor<0,001) mostra que a variável relaciona-se positivamente, na ordem de $216 \%$, com a opção do discente por adotar meios instrumentais (superficiais) para compreensão do assunto. Assim, as hipóteses nulas para $\mathrm{H7a}$ e $\mathrm{H7b}$ são rejeitadas.

Alguns pontos chaves são analisados diante dos achados desse estudo. Em H1b, Guimarães e Bzuneck (2008) defendem que a desmotivação caracteriza-se pela falta de interesse de quem realiza uma atividade. Entende-se que esse comportamento fomenta a realização de tarefas a fim de atingir um fim instrumental (Ryan \& Deci, 2000a; Biggs et al., 2001). No entanto, os resultados desse estudo não confirmam a relação positiva esperada entre desmotivação e estratégias instrumentais. Pesquisas como Boruchovitch e Bzuneck (2004), Guimarães e Bzuneck (2008) e Genc e Tinmaz (2013) defendem que, a motivação intrínseca está vinculada ao estímulo interno de realização de uma tarefa, fato que levaria o aluno a busca pela compreensão da causa em estudo, e não ao objetivo de obter um resultado (nota/média). Os achados para as H2a e H2b vão ao encontro das pesquisas supracitadas. 
A motivação introjetada é entendida como o estímulo de uma ação pelo sentimento de culpa, ameaça de punição ou promessa de recompensa por meio de fatores externos. Assim, acredita-se que a sensação gerada por esse tipo de pressão motiva os indivíduos a agirem de modo operacional, pois a crença não condiz com seus princípios pessoais. Os achados de $\mathrm{H} 3 \mathrm{a}$ e H3b ratificam as discussões realizadas sobre esse assunto (Deci \& Ryan, 1985; Guimarães \& Bzuneck, 2008). A quarta hipótese testada apresentou achados divergentes do esperado. Por ser considerada como um tipo de motivação extrínseca internalizada (Vansteenkiste et al., 2004b), esperava-se que a motivação identificada apresentaria uma relação positiva com o conhecimento aprofundado e negativa com as estratégias instrumentais. No entanto, os resultados comportaram-se de forma inversa ao esperado. Embora exista a concepção de que o comportamento é pessoalmente valioso, essa variável ainda é derivada de fatores externos, o que, por sua vez, estimulam comportamentos operacionais (Biggs et al., 2001; Genc \& Tinmaz, 2013).

Em H5, testou-se a motivação em que o indivíduo avalia a situação e coloca-a em congruência com seus valores e interesses pessoais, a regulação integrada. Embora represente a forma de motivação extrínseca mais avançada, e por vezes autodeterminada, a mesma ainda é considerada como externa (Gagné \& Deci, 2005). Os achados da pesquisa mostraram que esse tipo de regulação refletiu negativamente nas estratégias de estudo aprofundado dos alunos, conforme preconizado pela literatura (Vansteenkiste et al., 2004a; Kolb \& Kolb, 2005; Ferreira, 2009). Assim, os resultados parecem mostrar que comportamentos motivados extrinsicamente são realizados a fim de atingir um fim instrumental (Ryan \& Deci, 2000a; Genc \& Tinmaz, 2013).

O estudo analisa também as relações entre a regulação externa por frequência às aulas e as estratégias de aprendizagem. Os achados de H6 mostram resultados interessantes, sobretudo em H6a. A relação de 150\% dessa variável com a aprendizagem aprofundada diverge do esperado para uma motivação extrínseca (Biggs et al, 2001; Munshi et al., 2012). Diante desse fato, os alunos parecem entender que frequentar as aulas estimula a busca pela compreensão a essência do conteúdo, levando-os a adotar métodos de compreensão do assunto apresentado. Por fim, a variável de regulação externa por pressões sociais ratifica hipóteses anteriores acerca das associações entre as motivações extrínsecas e o envolvimento do indivíduo em uma tarefa (Vansteenkiste et al., 2004a; Ferreira, 2009; Kusurkar et al., 2012; Carmo, 2014). Acredita-se que a pressão que a sociedade exerce sobre o aluno o faz empregar meios alternativos, por vezes operacionais, para atingir o objetivo de atingir médias e/ou ser aprovado para um próximo período.

\section{CONSIDERAÇÕES FINAIS}

O estudo buscou verificar o efeito da motivação sobre as estratégias de aprendizagem adotadas pelos acadêmicos de Ciências Contábeis de uma universidade localizada no Estado do Paraná. Percebe-se que o número de mulheres estudantes em contabilidade é maior, representam $62,27 \%$ dos alunos, enquanto o sexo masculino corresponde a $37,73 \%$. Os respondentes tiveram maior concentração nos períodos iniciais. Em relação a idade, percebe-se que a maioria dos estudantes são jovens entre 18 e 23 anos, fato que corresponde a 87,76\% da população estudada. Apesar do número de mulheres ser maior, não foi encontrada relação significativa entre a motivação dos acadêmicos e as estratégias de aprendizagem adotadas por eles quando controlado pelo gênero.

Conforme os resultados, a motivação explica $61,55 \%$ da variação total das estratégias de estudo adotadas pelos alunos. Conforme a literatura, espera-se que níveis desmotivacionais seriam atrelados a falta de interesse pelo conteúdo, fato que estimularia o acadêmico a adotar meios operacionais para assimilar o conhecimento. Semelhante, a motivação extrínseca, estaria relacionada de forma semelhante com a estratégia de aprendizagem do aluno, estimulando-o a adotar meios instrumentais para alcançar seus objetivos. Por outro lado, a motivação intrínseca, fomenta o emprego de metodologias de aprendizagem fundamentadas, que levariam o aluno a compreensão de causa do conteúdo estudado (Boruchovitch \& Bzuneck, 2004; Gagné \& Deci, 2005; Guimarães \& Bzuneck, 2008).

Os achados ratificam, em grande parte, as hipóteses teóricas formuladas dos estudos tidos como base (Biggs et al., 2001, Guimarães \& Bzuneck, 2008, Kusurkar et al., 2012, Genc \& Tinmaz, 2013). Observou-se que os alunos que se mostram orientados à uma estratégia de aprendizado mais instrumental são positivamente associados as motivações/ regulações externas. Por outro lado, esses estímulos relacionam-se de forma negativa sobre a busca por conhecimento aprofundado.

Os resultados mostraram que aqueles que dedicam-se a um conhecimento aprofundado são positivamente movidos por estímulos internos, vontade própria de aprender, satisfação pessoal que a atividade gera. Em contrapartida, a motivação interna associou-se negativamente a opção pelo estudo de modo instrumental. A variável de desmotivação, por sua vez, mostrou que a relação foi negativa para ambas metodologias de estudo/aprendizagem. Entende-se que falta de proatividade e/ou interesse para cursar as disciplinas gera menor entusiasmo para compreensão das questões inerentes ao conteúdo transmitido, seja esta de modo aprofundado ou superficial.

Conclui-se que a motivação dos estudantes relaciona-se com as estratégias adotadas por estes, e que os estudantes orientados para uma estratégia mais instrumental são regulados por motivações externas, já com relação as estratégias de estudo aprofundanda esses estímulos tem vínculos negativos sobre o aprendizado. Com base nos resultados apresentados, os tipos motivacionais podem ter distintas relações com o desempenho acadêmico, dependendo da área de conhecimento, no caso da área de contabilidade, ela é marcada por vários estereótipos típicos da profissão (Leal et al., 2014). 
Este estudo oferece oportunidades de investigação aos interessados em compreender melhor os elementos principais de aprendizagem dos acadêmicos. Além da limitação espacial, a pesquisa foi realizada com acadêmicos de um dos câmpus universitários, fato que não permite generalizar o comportamento para toda instituição. Acredita-se que a comparação entre regiões e o período do curso pode apresentar resultados interessantes para futuras discussões. Ademais, o período de coleta de dados realizou-se no final do ano letivo e essa característica pode também ser significativa em outros momentos do curso. Por fim, além do gênero dos respondentes ser discutido como um elemento significativo em relação a estratégia de estudo, o período do curso, a função profissional, a participação em iniciação científica, entre outras variáveis, podem ser adicionadas no modelo como uma variável de controle.

\section{REFERÊNCIAS}

Biggs, J., Kember, D. \& Leung, D. Y. P. (2001). The revised two-factor Study Process Questionnaire: R-SPQ-2F. British Journal of Educational Psychology, 71, (pp. 133-149).

Boruchovitch, E. \& Bzuneck, J. A. (2004). A motivação do aluno: contribuições da psicologia contemporânea. 3. ed. Petrópolis: Vozes.

DeCharms, R. (1984). Personal causation: The internal affective determinants of behavior. In: R. E. Ames, \& C. Ames (Eds). Research on motivation in education. New York: Academic Press, Inc.

Deci, E. L. \& Ryan, R. M. (1985). Intrinsic motivation and self-determination in human behavior. New York: Plenum Press.

Deci, E. L. \& Ryan, R. M. (1991). A motivational approach to self: Integration in personality. In R. Dienstbier (Ed.), Nebraska symposium on motivation: Perspectives on motivation, 38, (pp. 237-288). Lincoln: University of Nebraska Press.

Fávero, L. P., Belfiore, P., Silva, F. L. \& Chan, B. L. (2009). Análise de dados: modelagem multivariada para tomada de decisões. Rio de Janeiro: Elsevier

Ferreira, M. (2009). Determinantes do desempenho acadêmico no ensino superior. Revista Internacional d'Humanitats, São Paulo/Barcelona

Gagné, M. \& Deci, E. L. (2005). Self-determination theory and work motivation. Journal of Organizational Behavior, 26,331-362.

Guimarães, S, Bzuneck, J. A. \& Sanches, S. F. (2002). Psicologia educacional nos cursos de licenciatura: a motivação dos estudantes. Psicologia Escolar e Educacional, 6,11-19.

Guimarães, S. E. R. \& Bzuneck, J. A. (2008). Propriedades psicométricas de um instrumento para avaliação da motivação de universitários. Ciências e Cognição, 13(1),101-113. Bookman.

Hair Jr., J. F., Black, W. C., Babin, B. J., Anderson, R. E. \& Tathan, R. L. (2009). Análise Multivariada de dados. 6.ed. São Paulo:

Harter, S. (1981) A new self-report scale of intrinsic vs. extrinsic orientation in the classroom: Motivational and informational components. Developmental Psychology, 17,300-312.

Kolb, A.Y. \& Kolb, D.A. (2005). Learning styles and learning spaces: enhancing experiential learning in higher education. AMLE, 4(2),193-212.

Kusurkar, R. A., Ten Cate, Th. J., Vos, C. M. P., Westers, P. \& Croiset, G. (2012). How motivation affects academic performance: a structural equation modelling analysis. Advances in Health Sciences Education, 18(1),57-69.

Leal, E. A., Miranda G. J. \& Carmo C. R. S. (2013). Teoria da Autodeterminação: uma Análise da Motivação dos Estudantes do Curso de Ciências Contábeis. Contabilidade \& Finanças, 24(62),162-173.

Leal, E. A., Miranda G. J., Araujo, T. S. \& Borges, L. F. M. (2014). Estereótipos na Profissão Contábil: a opinião de estudantes e do público externo no Triângulo Mineiro. Contabilidade, Gestão e Governança, 17(1),134-153.

Lens, W., Matos, L. \& Vansteenkiste, M. (2008). Professores como fontes de motivação dos alunos: o quê e o porquê da aprendizagem do aluno. Educação, 31(1),17-10.

Morin, E. A. (2004). Cabeça bem-feita: repensar a reforma, reformar o pensamento. Rio de Janeiro: Bertrand Brasil.

Munshi, F. M., Al-Rukban, M. O. \& Al-Hoqail, I. (2012). Reliability and validity of an Arabic version of the revised two-factor study process questionnaire R-SPQ-2F. Journal of Family and Community Medicine, 19(1),33-37.

Reeve, J., Deci, E. L. \& Ryan, R. M. (2004). Self-determination theory. A dialectical framework for understand sociocultural influences on student motivation. In: McInerney D. M. \& Van Etten S. (Eds.) Big Theories Revisited, (pp. 31-58). Connecticut: Age Publishing.

Ryan, R. M. \& Deci, E. L. (2000a). Selfdetermination theory and the facilitation of intrinsic motivation, social development, and well-being. American Psychologist, 55(1),68-78.

Ryan, R. M. \& Deci, E. L. (2000b). Intrinsic and Extrinsic Motivations: Classic Definitions and New Directions. Contemporary Educational Psychology, 25,54-67.

Vansteenkiste, M., Simons, J., Lens, W., Sheldon, K. M. \& Deci, E. L. (2004a). Motivating Learning, Performance, and Persistence: The Synergistic Effects of Intrinsic Goal Contents and Autonomy-Supportive Contexts. Journal of Personality and Social Psychology, 87(2),246-260.

Vansteenkiste, M., Lens, W., De Witte, S., De Witte, H. \& Deci, E. L. (2004b). The 'why' and 'why not' of job search behavior: Their relation to searching, unemployment experience, and well-being. European Journal of Social Psychology, 34,345-363. 
Vansteenkiste, M., Zhou, M., Lens, W. \& Soenens, B. (2005). Experiences of autonomy and control among Chinese learners: Vitalizing or immobilizing? Journal of Educational Psychology, 97(3),468-483.

Wanous, M., Procter, B. \& Murshid, K. (2009). Assessment for learning and skills development: The case of large classes. European Journal of Engineering Education, 34(1),77-85.

White, W. R. (1975). Motivation reconsidered: the concept of competence. In Mussem P. H., Conger J. J., \& Kagan J. (Orgs.), Basic and contemporary issues in developmental psychology (pp. 266-230). New York: Harper \& Row. 